\title{
Frequency comb based absolute frequency reference design for future spaceborne multi species differential absorption lidar systems for green- house gases monitoring
}

D. C. Heinecke, D. Fehrenbacher, L. Nagy, A. Baatzsch, M. Herding, et al.

D. C. Heinecke, D. Fehrenbacher, L. Nagy, A. Baatzsch, M. Herding, J. B. Dherbecourt, R. Santagata, M. Raybaut, H. Schäfer, "Frequency comb based absolute frequency reference design for future spaceborne multi species differential absorption lidar systems for green-house gases monitoring," Proc. SPIE 11852, International Conference on Space Optics — ICSO 2020, 118526D (11 June 2021); doi: 10.1117/12.2600288

SPIE Event: International Conference on Space Optics - ICSO 2021, 2021, Online Only 


\section{International Conference on Space Optics-ICSO 2020}

Virtual Conference

30 March-2 April 2021

Edited by Bruno Cugny, Zoran Sodnik, and Nikos Karafolas
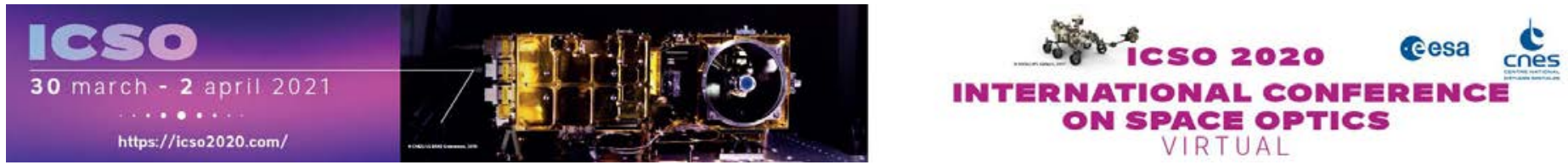

Frequency comb based absolute frequency reference design for future spaceborne multi species differential absorption lidar systems for green-house gases monitoring

\section{Cesa issopocestings denes}




\title{
Frequency comb based absolute frequency reference design for future spaceborne multi species differential absorption lidar systems for green-house gases monitoring
}

\author{
D. C. Heinecke ${ }^{1 *}$, D. Fehrenbacher ${ }^{1}$, L. Nagy ${ }^{1}$, A. Baatzsch ${ }^{1}$, M. Herding ${ }^{1}$, J.B. Dherbecourt ${ }^{2}$, \\ R. Santagata ${ }^{2}$, M. Raybaut ${ }^{2}$, H. Schäfer ${ }^{1}$ \\ ${ }^{1}$ SpaceTech GmbH, Seelbachstr. 13, 88090 Immenstaad, Germany, ${ }^{2}$ DPHY, ONERA, Université \\ Paris Saclay, F-91123 Palaiseau, France \\ * Corresponding author: dirk.heinecke@spacetech-i.com
}

\begin{abstract}
The Lidar Emitter and Multi-species greenhouse gases Observation iNstrument (LEMON) is a novel Differential Absorption Lidar (DIAL) sensor concept for greenhouse gases and water vapor measurements from space..$^{1,2}$ It is based on a versatile transmitter allowing for addressing various absorption lines of different molecules. This highly flexible emitter design requires a universal frequency referencing scheme. Here we present a concept employing a $1 \mathrm{GHz}$ frequency comb, which allows the absolute referencing over a spectral range from $0.95 \mu \mathrm{m}$ to $1.15 \mu \mathrm{m}$. By using an intermediate frequency doubling stage, this allows for DIAL measurements on $\mathrm{CO}_{2}, \mathrm{H}_{2} \mathrm{O} / \mathrm{HDO}$, and $\mathrm{CH}_{4}$ in the $2 \mu \mathrm{m}$ range. Absolute referencing is obtained by using a GPS disciplined oscillator as the common time base for frequency measurements. The concept of the LEMON Frequency Reference UnIT (FRUIT) is designed to match the requirements of the vibration loads associated with airborne operation to allow implementation on the airborne demonstrator for LEMON. In addition, the requirements for a future space development are considered in the design. For example, radiation critical items have been identified and radiation tested within the project and a compact wavemeter design has been implemented.
\end{abstract}

Keywords: LIDAR, greenhouse gas, DIAL, frequency reference, frequency comb

\section{INTRODUCTION}

The LEMON instrument shall be implemented on an airborne platform as a demonstrator for the maturity of the technique w.r.t a spaceborne implementation. For that reason, the requirements somewhat depend on the application platform. In the present project stage with an airborne demonstrator as a hardware deliverable, the airborne requirements are respected for the hardware implementation, while the requirements for the spaceborne instrument are either analysed or tested within dedicated space assessment tests.

Performance requirements

The performance requirements are derived from the spectroscopic needs. To have optimal signal for the integrated path DIAL measurement, the online wavelength needs to be well located on the edge of the absorption line to provide enough return signal on the one hand and gas specific absorption sensitivity on the other hand. This leads to a requirement for the frequency accuracy and stability of $1 \mathrm{MHz}$. Both, the accuracy (mean value) and the stability (RMS value) shall be achieved with the statistical set acquired during any 100 second time window. In order to cover the different species $\left(\mathrm{H}_{2} \mathrm{O}, \mathrm{HDO}\right.$, $\mathrm{CO}_{2}$ ) this requires to meet the above accuracy at different wavelengths in the spectral range from $1982 \mathrm{~nm}$ to $2051 \mathrm{~nm}$. For $\mathrm{CH}_{4}$ the online frequency is located around $2290 \mathrm{~nm}$, see Figure 1. More details are covered in the ICSO 2020 contribution on the overall LEMON instrument design: P050 "Design and pre-development of an airborne multi-species differential absorption Lidar system for water vapor and HDO isotope, carbon dioxide, and methane observation". ${ }^{2}$

In order to achieve this high accuracy over such a large spectral band, it was decided to use a frequency comb as a reference that covers the entire spectral region. The design foresees to achieve the absolute frequency of the laser light by the following measures:

1.) The optical parametric amplifier configuration (crystal parameters, temperature tuning) in the pulsed main laser emitter determines the frequency of the laser pulses that are used for the spectroscopy. This configuration leads 
to a knowledge of the laser frequency with an accuracy of about $10 \mathrm{~nm}$, corresponding to $5 \mathrm{~nm}$ for the frequency doubled light.

2.) A two-element wavemeter is used to retrieve the absolute frequency with accuracies of $<4 \mathrm{GHz}$ (coarse level) and $<10 \mathrm{MHz}$ (fine level)

3.) Finally, a beating against the frequency comb retrieves the absolute frequency with an accuracy of $100 \mathrm{kHz}$ with a goal of $10 \mathrm{kHz}$ for the spaceborne FRUIT.

For technical reasons, the frequency metrology is done at the frequency doubled wavelength (around $1 \mu \mathrm{m}$ ) where silicon sensors can be used. In principle, beating detection at the fundamental wavelength around $2 \mu \mathrm{m}$ is also possible.

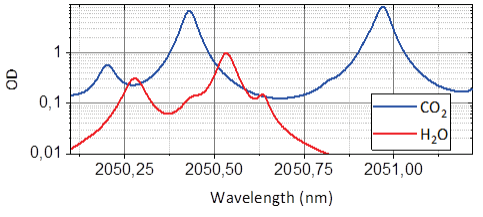

(a)

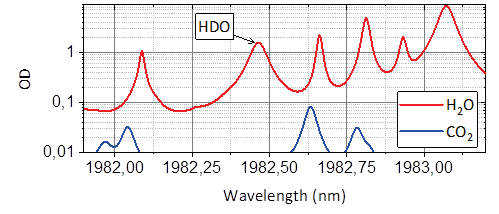

(b)

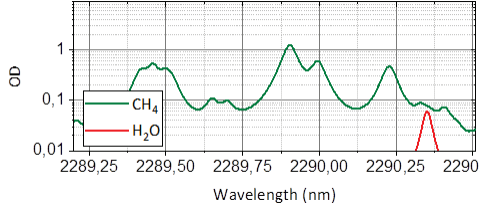

(c)

Figure 1. Optical depth calculated within the three main spectral regions of interest with HITRAN 2016 for a $6 \mathrm{~km}$ vertical path (from ground). $\mathrm{CO}_{2}$ absorption band (a), water vapor and $\mathrm{HDO}$ absorption band (b), $\mathrm{CH}_{4}$ absorption band (c).

\section{Environmental requirements}

The airborne instrument is subjected to variations in temperature and pressure during operation. In addition, continuous acoustic and mechanical vibrations will impact on the system. Therefore, hermetic housings have been implemented for those devices that are sensitive to pressure changes. Damping systems shall decouple the system from mechanical loads and where needed a temperature stabilization is foreseen to compensate for drifts.

In contrast, the spaceborne system will have a single change in pressure but constant vacuum conditions during operation. Also, vibrational and acoustic loads are only present during the space launch but not during operation. Given the vacuum environment, thermal changes are rather slow and can be easily compensated by temperature stabilization measures. On the other hand, radiation will impact the electrical and electro-optical components. Therefore, at the present project stage, the space activities are mainly focusing on the radiation topic. Some details are provided in section 3. Furthermore, vibration tests are foreseen to prove the mechanical stability of the most sensitive free space optical components of the main laser. ${ }^{2}$ For the wavemeter, the heritage design of the MERLIN frequency reference unit has been brought to the new evolution step. ${ }^{3}$

\section{SYSTEM DESIGN AND IMPLEMENTATION}

The frequency ruler, which the FRUIT employs to measure absolute frequencies, is based on a frequency comb. The frequency comb acts as a transfer oscillator to transfer the stability and accuracy of a GPS disciplined oscillator into the optical domain. For now more than 20 years, the concept of using frequency combs for bidirectional transfer between the radio-frequency and optical domains is well established, ${ }^{4}$ in particular in the scientific lab environment for atomic and optical clock comparisons..$^{5}$ Applications of frequency combs in the field and outside the lab ${ }^{6,7}$ as well as their use as frequency references for lidar systems ${ }^{8}$ only start to emerge. Wavelength conversion or expansion is intrinsically required for combs that are using a f-2f interferometer for stabilization. Due to ultra-short pulse duration and high peak powers associated with the mode-locked laser producing the frequency comb, nonlinear wavelength conversion in bulk, and more efficiently in fibres or waveguides is possible. Thinking towards a spaceborne system the complexity should be reduced as much as possible. Therefore, we decided to investigate a system design without employing a transfer laser, measuring the transmitter pulses with heterodyne beat frequency detection directly with individual comb lines. This requires a wide spaced frequency comb given the transmitter linewidth and free-spectral range. A femtosecond laser with a repetition rate of $1 \mathrm{GHz}$ operating at a wavelength of $1.5 \mu \mathrm{m}$ was chosen. The laser provides the performance allowing for frequency comb stabilization, ${ }^{9}$ the required large comb mode spacing, and it is constructed to withstand the loads associated with operation in an airplane. When heterodyning an optical signal against one teeth of a frequency comb, the index number of the comb mode needs to be known in order to obtain an absolute frequency value. One method to determine this mode 
index is the use of a low resolution, but calibrated auxiliary wavemeter, to make a coarse measurement of the wavelength of the optical signal. The resolution of this measurement needs to be better than the comb mode spacing or repetition rate of the frequency comb. Hereby, a frequency comb with a repetition rate in the GHz range is of benefit as well, since it relaxes the resolution requirement of the wavemeter.

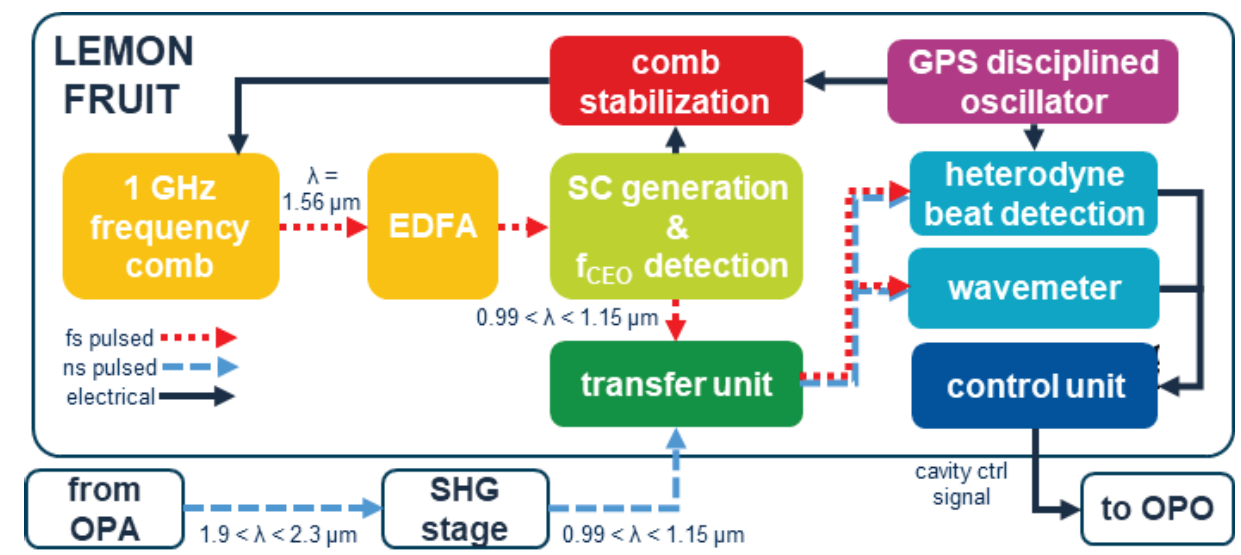

Figure 2. Schematic of the LEMON FRUIT. The following abbreviations are used. EDFA: erbium-doped fibre amplifier, SC: supercontinuum, OPA: optical parametric amplifier, OPO: optical parametric oscillator, SHG: second harmonic generation.

Figure 2 shows a schematic of the FRUIT system. The output of the laser is amplified in an erbium-doped fibre amplifier (EDFA). After dispersion compensation, a supercontinuum (SC) spanning an octave and covering the lidar spectral range is generated in a highly nonlinear fibre (HNF). One part of the supercontinuum is split off for detecting the carrier envelope offset frequency of the comb. After stabilization of the two degrees of freedom of the comb, the frequency of the comb modes is fixed and known. The second part of the supercontinuum is filtered and spatially and temporally overlapped with the light from the emitter. The $2 \mu \mathrm{m}$ OPA pulses are frequency doubled (SHG: second harmonic generation) to shift the detection to $1 \mu \mathrm{m}$. With a fast analogue-to-digital converter (ADC) the heterodyne beat signal between comb mode and SHG pulses is sampled. ${ }^{10}$ Together with a low-resolution wavemeter measurement the control unit can determine the absolute frequency of the SHG pulse. The obtained frequency value is used for lidar data analysis, but also for providing an error signal for active stabilization of the lidar laser emitter oscillator.

\section{Supercontinuum generation}

Supercontinuum generation serves two purposes. The first one is to convert spectral power from the seed laser at a wavelength of $1550 \mathrm{~nm}$ to the frequency region of the SHG of the absorption lines of the relevant greenhouse gases. The second purpose is the generation of coherent radiation needed for employment of an $\mathrm{f}-2 \mathrm{f}$ interferometer, see next paragraph. At best, both tasks are fulfilled simultaneously without the need of changing system parameter (e.g. pump power of the amplifier). An overview of the spectral parts of interest and the implementation of the LEMON FRUIT is displayed in Figure 3. The power spectral density of the seed, the amplifier and the generated supercontinuum are shown on a logarithmic scale. In addition, the wavelengths to be covered for beating with the OPO are highlighted. The range of the latter extends from $990 \mathrm{~nm}$ to $1145 \mathrm{~nm}$. Hence, for the f-2f interferometer spectral parts in the range of $1980 \mathrm{~nm}-2190 \mathrm{~nm}$ are needed. This is achieved by boosting the pulse energy of the seed laser (centered at $1550 \mathrm{~nm}$ ) with a commercial amplifier to a level exceeding $1.5 \mathrm{~nJ}$. At this stage the pulses are spectrally broadened due to nonlinear effects, namely self-phase modulation. In order to obtain short pulses at the end of the amplifier a patch chord of dispersion compensating fibre has been inserted between the seed laser and the amplifier. The output of the amplifier is connected to the HNF and a dispersive wave centered at roughly $1010 \mathrm{~nm}$ and a soliton around $2150 \mathrm{~nm}$ is emerging. To guarantee best performance and interference-free operation, the connections between the modules are implemented with fibre connectors and all used fibres including the HNF are of type polarization-maintaining single mode bulk fibres. Due to different mode field diameters between HNF and dispersion compensating fibres, low loss splices to connectorized standard PM single mode fibres are used for interfacing. 


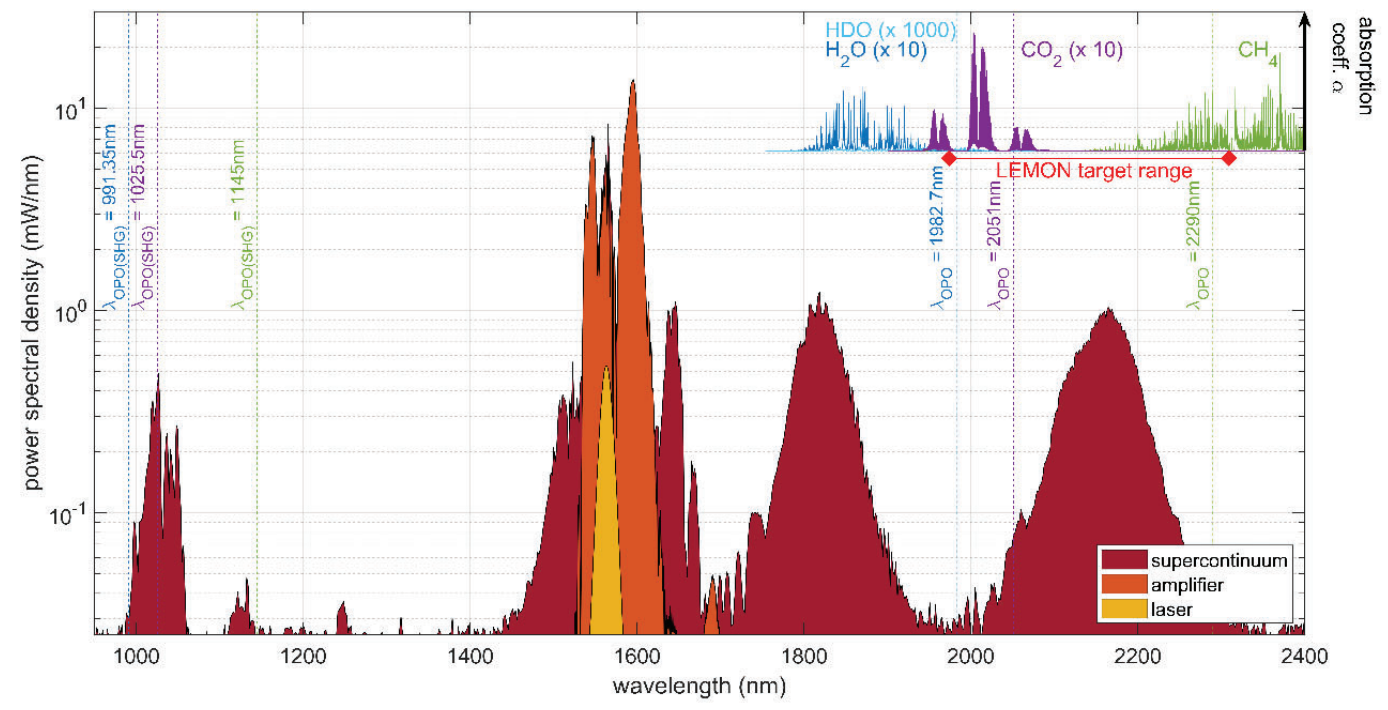

Figure 3. Spectrum of the seed laser, amplifier and the supercontinuum together with the frequencies of the absorption lines to be measured.

\section{Nonlinear interferometer}

In order to absolutely stabilize the frequency comb, both degrees of freedom of the comb have to be stabilized. Namely, the comb spacing associated with the repetition rate $\left(f_{\text {rep }}\right)$ of the laser and the comb shifting associated with the carrierenvelope offset frequency $\left(f_{\mathrm{CEO}}\right)$ variation, respectively. While the former is directly accessible by measuring the repetition rate of the pulsed laser source, the latter needs some absolute reference at least at one optical frequency. This could be an optical absorption of a molecule or atom. In our case, we make use of the so called self-referencing scheme, ${ }^{11}$ which allows us to stabilize the optical comb shifting degree with the help of the GPS disciplined oscillator source that serves as our absolute reference.

In our system the self-referencing is done at the same wavelength range as we do the frequency metrology of the lidar laser that is stabilized by the LEMON FRUIT. Therefore, some part of the octave spanning frequency comb light at $2 \mu \mathrm{m}$ is frequency doubled to $1 \mu \mathrm{m}$ where it is overlapped in time, space and polarization with the directly generated spectral parts at of the supercontinuum at $1 \mu \mathrm{m}$. This signal is focused on a photo diode to measure the beat note between the two signals which corresponds the offset frequency $\left(\mathrm{f}_{0}\right)$ of the comb - also named and known as carrier-envelope offset frequency $\left(\mathrm{f}_{\mathrm{CEO}}\right)$ in other areas of ultrafast lasers. In addition, the repetition rate signal is measured with the same photo diode (bandwidth greater than $1 \mathrm{GHz}$ ), see also section 3. The nonlinear interferometer is a critical subunit with respect to environmental loads and therefore a robust opto-mechanical design is needed. For the airborne implementation a hybrid design based on COTS and custom parts (mostly stainless steel) to fulfil mechanical and thermal stability requirements has been developed, see Figure 4. 


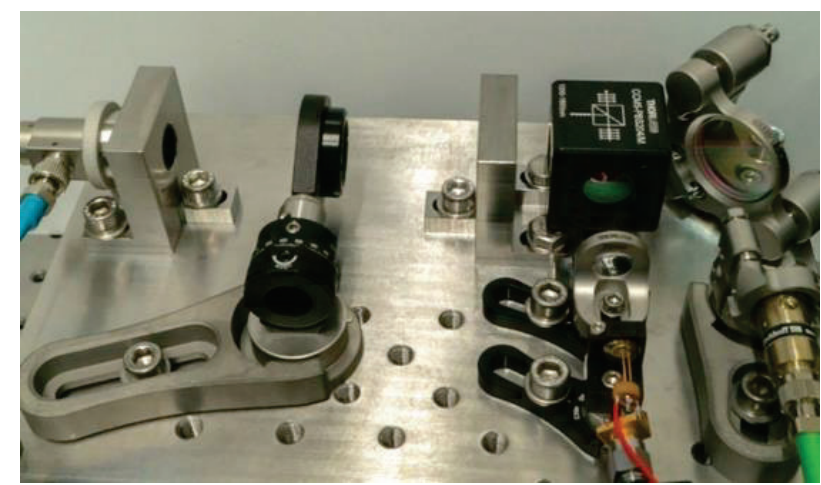

Figure 4. Photograph of the nonlinear interferometer optical bench for the airborne instrumentation.

\section{Wavemeter}

While the frequency comb provides a very precise absolute reference, its ambiguity defined by the repetition rate requires additional spectroscopic tools to determine the comb mode index of the mode that is used for frequency metrology. In our case, the $1 \mathrm{GHz}$ repetition rate leads to an ambiguity as a frequency difference between the comb mode and the measured signal of e.g. $200 \mathrm{MHz}$ and $800 \mathrm{MHz}$ (equal to $-200 \mathrm{MHz}$ to the next comb mode) will lead to the same beating signal. For resolving this issue, a wavemeter is needed that allows at least for an absolute accuracy of $<250 \mathrm{MHz}$. With a nominal beating offset frequency of $250 \pm 125 \mathrm{MHz}$, the wavemeter is able to

1. determine the relative position of the comb mode (e.g. $-250 \mathrm{MHz}$ or $+250 \mathrm{MHz}$ )

2. determine the comb mode index.

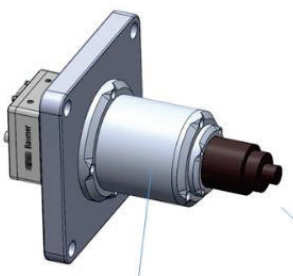

Camera

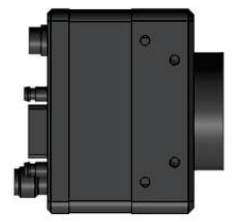

Fizeau assembly

Collimator
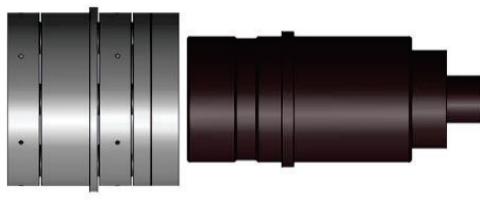

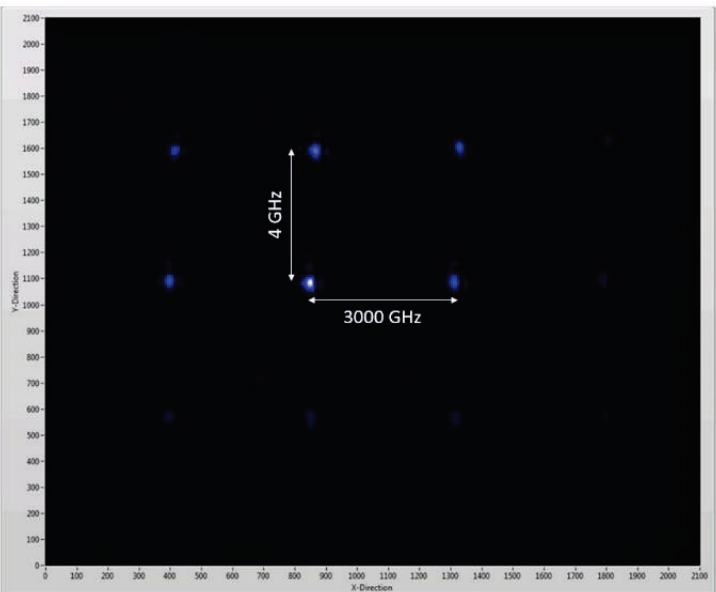

Figure 5. (left) CAD Model of the wavemeter design. (right) Intensity pattern of a single mode laser source on the CCDcamera of the 2-D-wavemeter, where the horizontal dimension corresponds to the coarse and the vertical dimension to the fine resolution.

On the other side, the parameter adjustment of the optical parametric oscillator in the pulsed laser emitter of the lidar instrument defines the absolute frequency, respectively wavelength, with an accuracy of $10 \mathrm{~nm}$ at $2 \mu \mathrm{m}$. For the frequency doubled light at $1 \mu \mathrm{m}$, this corresponds to an absolute accuracy of $5 \mathrm{~nm}$ or $1500 \mathrm{GHz}$.

Therefore, the wavemeter needs to determine the absolute frequency with an accuracy of $<250 \mathrm{MHz}$ while spanning a spectral window of $1500 \mathrm{GHz}$ unambiguously. This requires a relative resolution of about $1.6 \cdot 10^{-4}$ which is rather 
challenging for a wavemeter equipped with a single Fizeau wedge. Therefore, a wavemeter design with two Fizeau wedges was chosen in order to achieve the requirements with sufficient margin.

The baseline specifications of the wavemeter are:

- Coarse dimension:

- Fine dimension:
$3000 \mathrm{GHz}(\sim 10 \mathrm{~nm})$ free spectral range

$4 \mathrm{GHz}$ free spectral range

This relaxes the resolution requirement to $4 / 3000 \approx 0.1 \%$ for the coarse dimension while in the fine dimension it is even more relaxed to about $6 \%$ resolution. The reason for not choosing a higher FSR in the fine dimension was to have an FSR that is a multiple of the repetition rate of the laser comb. This enables the option to calibrate the wavemeter function (space versus frequency) with the help of the frequency comb. If the FSR is chosen too high, the single comb modes cannot be resolved anymore. For implementation of a spaceborne system, also size and weight are driving factors. This has been taken into account for the wavemeter design as well. Starting from the established flight design from the MERLIN FRU, ${ }^{3}$ we decided to implement a two dimensional wavemeter where two orthogonal Fizeau wedges are entangled in one compact optical block. On the left side of Figure 5 the arrangement of the main elements is shown. As can be seen, the whole wavemeter has a simple linear configuration, which allows for a robust mechanical embedding. On the right hand side of Figure 5, a sample of a measured fringe pattern generated by a single frequency laser source is shown.

Finally, the wavemeter design applies already for both airborne and spaceborne requirements. For the airborne implementation, the wavemeter will be incorporated into a hermetic housing that allows for constant pressure conditions and further supports the acoustic and thermal decoupling from the environment.

\section{RESULTS}

\section{Frequency comb stabilization}

The stabilization of the two degrees of freedom of the comb, repetition rate and offset frequency, is implemented as follows, see Figure 6. On the fast photodiode in the f-2f setup, both repetition rate and offset frequency are measured. The two signals are separated using a suitable RF diplexer. The repetition rate signal is fed into a phase-locked loop where it is compared against a $1 \mathrm{GHz}$ signal referenced to the GPSDO. The error signal is sent to a digital PID controller and applied to two intra-cavity PZTs to control the laser cavity length. The offset frequency signal is measured using a fast frequency counter and low bandwidth feedback $(<1 \mathrm{kHz})$ is applied to the laser via pump current modulation. Since the stability of the offset frequency is less critical as for the repetition rate, there is no need for a PLL. Figure 7 shows first measurement results on the achieved comb stability, the projection on the expected optical stability at $1 \mu \mathrm{m}$ and a comparison with the system requirements.
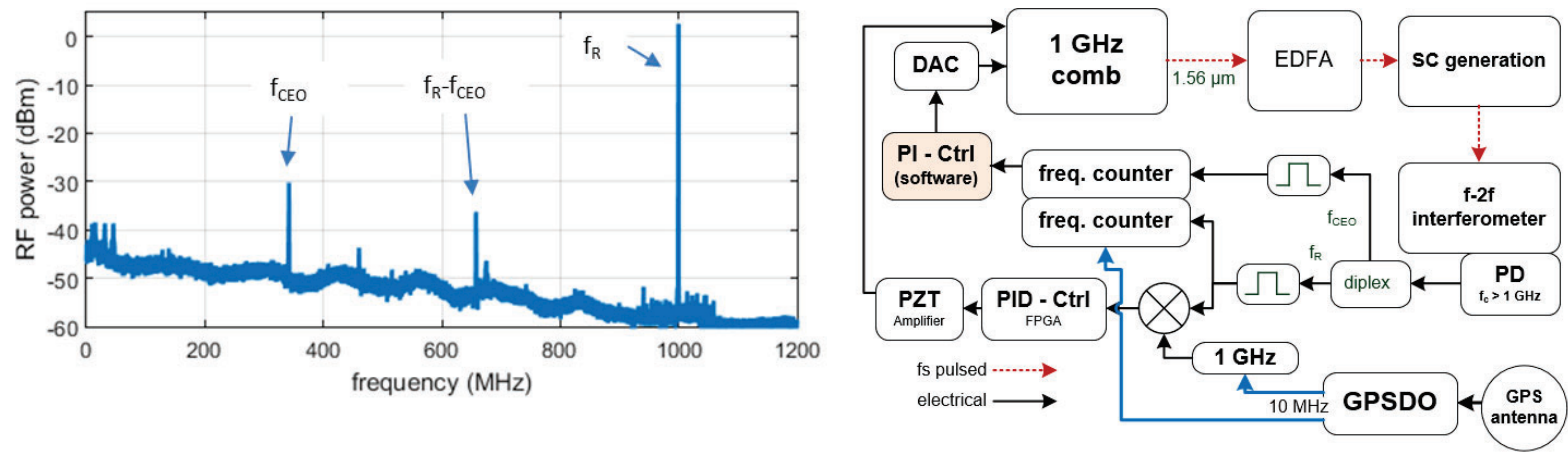

Figure 6. (left) RF spectrum measured after the photodiode of the nonlinear interferometer and (right) stabilization schematic. .Abbreviations used: EDFA: erbium-doped amplifier, SC: supercontinuum, PD: photodiode, GPSDO: GPS disciplined oscillator, PICtrl: Proportional-Integral Controller, PID-Ctrl: Proportional-Integral-Derivative Controller, PZT: Piezo-electric transducer, DAC: digital-to-analogue converter. 

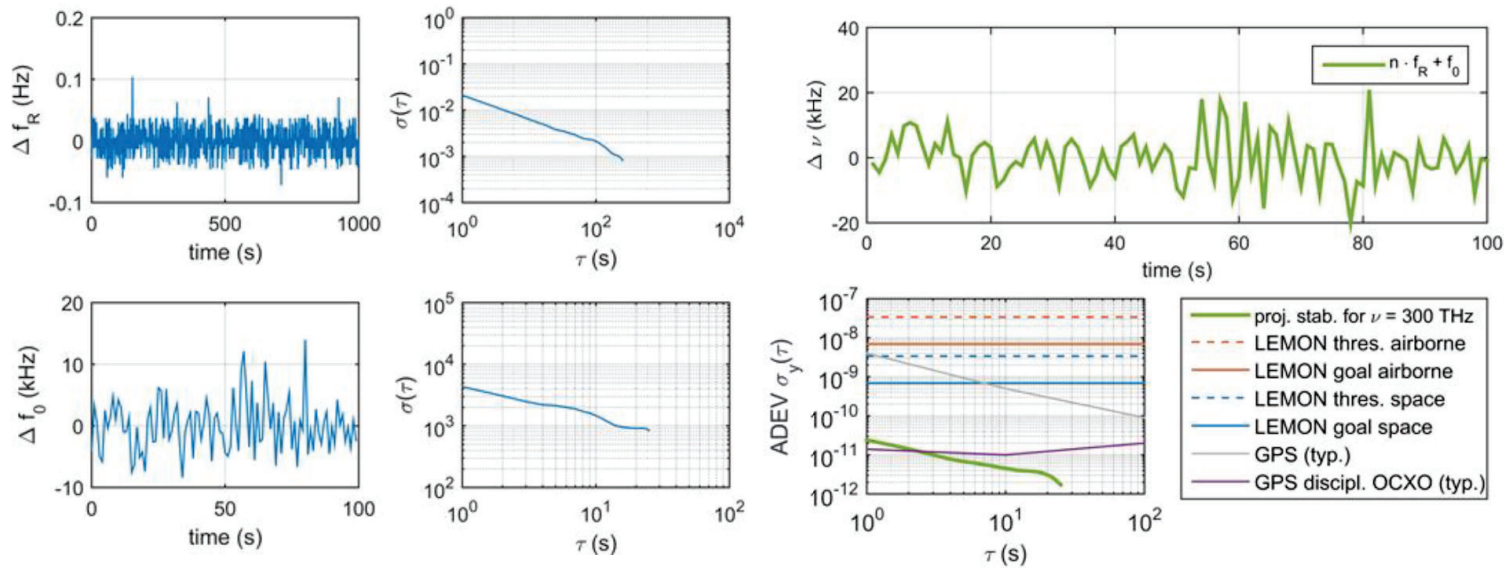

Figure 7. Frequency stability measurements and stability plot for the two degrees of freedom of the frequency comb. (Bottom right) measured stability in conjunction with LEMON stability requirements.

\section{Radiation testing of nonlinear fibre}

The fibres for supercontinuum generation are categorized as photo sensitive and hence the utilisation in a space environment is seen critical. However, due to the short length of the fibres the impact of radiation to their performance especially in a low earth environment typical for a LIDAR Mission might be negligible. To investigate the usability of the HNFs for such a mission, two different nonlinear fibres have been subjected to a radiation test with protons. The test has been conducted by ONERA in the framework of another test campaign at the UCL facility in Belgium. The test conditions are listed in Table 1 and are based on simulations of the environment of typical polar low earth missions of 5 years duration.

Table 1

\begin{tabular}{|c|c|c|c|}
\hline Energy of Protons $(\mathrm{MeV})$ & Fluency $\left(\mathrm{p}^{+} / \mathrm{cm}^{-2}\right)$ & Flux $\left(\mathrm{p}^{+} / \mathrm{cm}^{-2} . \mathrm{s}\right)$ & Equivalent TID (krad [Si]) \\
\hline 60 & $4 \times 10^{10}$ & $1 \times 10^{8}$ & 5.5 \\
\hline
\end{tabular}

Two different HNFs have been spliced to a fibre that was known to not be radiation sensitive at the doses listed above. Characterization of the test item before and after the testing is performed by comparing the supercontinuum generated from a fs-laser source at $1550 \mathrm{~nm}$. To ensure the measurements are not compromised by changes in the laser setup, a third HNF sample has been fabricated, but not irradiated. A graph of the supercontinuum of all three fibres before and after the radiation test is displayed in Figure 8. Note that the success criteria is the actual wavelength of the spectral components generated and not their intensity. The latter one is different on successive measurements due to non-uniform coupling of the spectral parts into the spectrometer. Before and after the radiation tests the spread of the supercontinuum remains constant for both tested fibres, as well as for the reference fibre. Hence, no radiation induced damage has been detectable on the two different HNFs investigated. 

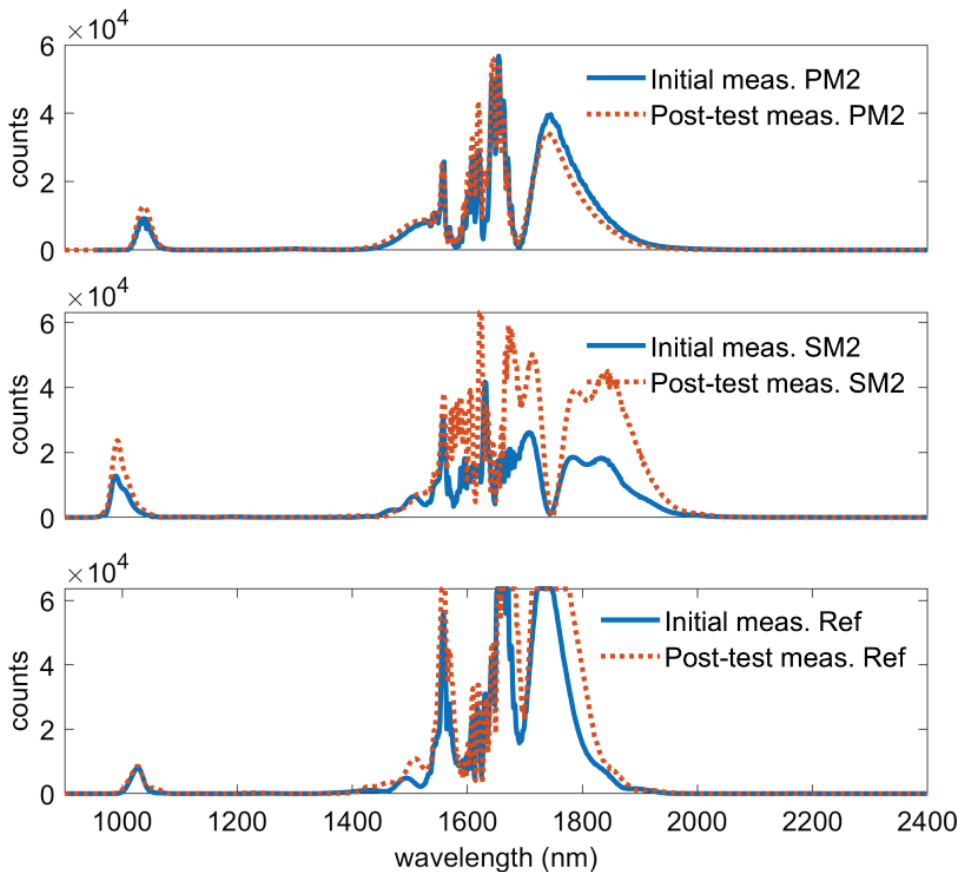

Figure 8. Radiation test results for the two different fibres. The fibre labelled as "Ref" has not been irradiated.

\section{SUMMARY}

In this paper we present results on design and implementation of a frequency comb based absolute frequency reference concept for future spaceborne multi-species differential absorption lidar systems for green-house gases monitoring. For demonstration purposes an airborne DIAL instrument is under development. The airborne FRUIT will be integrated in a 19 inch rack. Despite no specific optimization in terms of weight, size and power has been performed, the FRUIT will be relatively compact with a volume of about 1001 and a weight of less than $40 \mathrm{~kg}$. The power consumption of the system will be in the range of $200 \mathrm{~W}$. It is expected that volume, weight and power consumption can be reduced by a factor of ten for a spaceborne implementation. In addition to implementing the airborne demonstrator, the frequency reference unit concept has been evaluated successfully for its space suitability and radiation tests on components considered as radiation critical have been performed.

\section{Acknowledgments}

We would like to thank Dong Hui Wang of OFS and Zahir Hussain of Sumitomo for providing test samples.

This research has received funding from the European Union's Horizon 2020 research and innovation program under grant agreement $\mathrm{N}^{\circ} 821868$.

\section{REFERENCES}

[1] Lemon-dial-project.eu

[2] Dherbecourt et al., Design and pre-development of an airborne multi-species differential absorption Lidar system for water vapor and HDO isotope, carbon dioxide, and methane observation. ICSO 2020, P050 (2021).

[3] Schaefer et al., Flight design of the absolute frequency reference unit for the methane-sensing LIDAR Mission MERLIN, Proc.SPIE (2019) 111802L. 
[4] Fortier and Baumann, 20 years of developments in optical frequency comb technology and applications. Commun Phys 2, 153 (2019) https://doi.org/10.1038/s42005-019-0249-y.

[5] Hollberg et al., The measurement of optical frequencies, (2005), https://iopscience.iop.org/journal/0026-1394

[6] Coburn et al., Regional trace-gas source attribution using a field-deployed dual frequency comb spectrometer, Optica 5, 320-327 (2018).

[7] Ycas et al., Demonstration of on-sky calibration of astronomical spectra using a $25 \mathrm{GHz}$ near-IR laser frequency comb, Optics Express, Vol. 20, No. 6. (2012).

[8] Lezius et al., Space-borne frequency comb metrology, Optica 3, 1381-1387 (2016).

[9] Lind et al., Frequency-stabilized $1 \mathrm{GHz}$ turnkey frequency comb, in Laser Congress 2019 (ASSL, LAC, LS\&C), OSA Technical Digest (Optical Society of America, 2019), paper JTh3A.51.

[10] Wirth et al., The airborne multi-wavelength water vapor differential absorption lidar WALES: system design and performance, Appl. Phys. B (2009) 96: 201.

[11] Telle et al., Carrier-envelope offset phase control: A novel concept for absolute optical frequency measurements and ultrashort pulse generation, Appl. Phys. B (1999) 69: 327. 